\title{
Experience with the Norwood procedure without circulatory arrest
}

Yutaka Imoto, MDa

Hideaki Kado, MD

Yuichi Shiokawa, MDa

Kazu Minami, MDa

Hisataka Yasui, MD

From Cardiovascular Surgery, ${ }^{\mathrm{a}}$ Fukuoka Children's Hospital, and the Division of Cardiovascular Surgery, ${ }^{\mathrm{b}}$ Kyusyu University, Fukuoka, Japan.

Received for publication Oct 10, 2000; revisions requested Nov 6, 2000; revisions received April 2, 2001; accepted for publication April 30, 2001.

Address for reprints: Yutaka Imoto, MD, Cardiovascular Surgery, Kyushu KoseiNenkin Hospital, 2-1-1 Kishinoura, Yahatanishi-ku, Kitakyushu 806-8501, Japan.

J Thorac Cardiovasc Surg 2001;122:879-82

Copyright (C) 2001 by The American Association for Thoracic Surgery

$0022-5223 / 2001 \$ 35.00+0 \quad \mathbf{1 2 / 1 / 1 1 6 9 4 8}$

doi: $10.1067 / \mathrm{mtc} .2001 .116948$
Objective: We evaluated a new cardiopulmonary bypass technique that allowed complete avoidance of circulatory arrest and deep hypothermia in the Norwood procedure for hypoplastic left heart syndrome.

Methods: A total of 10 patients were included in this study. The arterial line of the cardiopulmonary bypass circuit was divided in two in a Y shape; one branch was used for cerebral perfusion through the innominate artery and the other for lower body perfusion through the cannula inserted into the descending thoracic aorta. Moderate hypothermia $\left(29^{\circ} \mathrm{C}-31^{\circ} \mathrm{C}\right.$ rectal temperature) and high pump flow (150$180 \mathrm{~mL} \cdot \mathrm{kg}^{-1} \cdot \mathrm{min}^{-1}$ ) were used. A valveless conduit between the right ventricle and the pulmonary artery was used in 6 patients as an alternative pulmonary blood source to a conventional Blalock-Taussig shunt $(n=4)$.

Results: Circulatory arrest was completely avoided throughout the operation in all cases, and no complications from the new cardiopulmonary bypass technique were seen. Early deaths occurred in 3 cases. Neurologic deficits were not seen among the survivors, and the postoperative course was stable and uneventful, including satisfactory renal function.

Conclusions: The Norwood procedure for hypoplastic left heart syndrome was successfully accomplished with complete avoidance of circulatory arrest by means of cerebral perfusion through the innominate artery combined with cannulation of the descending aorta. A conduit between the right ventricle and the pulmonary artery seems an excellent alternative pulmonary blood source, although right ventricular function needs to be carefully monitored.

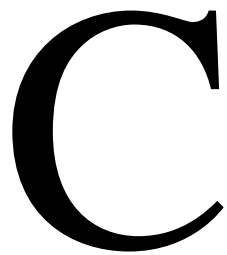

irculatory arrest with deep hypothermia is widely used for arch reconstruction in pediatric cardiac surgery including the Norwood procedure for hypoplastic left heart syndrome. However, potential risks of complications such as neurologic deficits and renal failure cannot be ignored. ${ }^{1-5}$ We developed a new cardiopulmonary bypass (CPB) technique that enabled us to avoid circulatory arrest throughout the operation. The bypass technique, operative procedure, and the results in the Norwood procedure are presented herein.

\section{Patients and Methods}

From June 1998 to September 1999, ten patients with hypoplastic left heart syndrome underwent the Norwood procedure (Table 1). Ages at operation ranged from 2 to 30 days (17.4 \pm 9.2 days) and body weights from 1.7 to $3.2 \mathrm{~kg}(2.6 \pm 0.4 \mathrm{~kg})$. The combination of aortic and mitral atresia was recognized in 7 patients, aortic atresia and mitral stenosis in 2 patients, and aortic and mitral stenosis in 1 patient. The CPB technique has been reported elsewhere. ${ }^{6}$ After median ster- 
TABLE 1. Summary of the cases

\begin{tabular}{rccccccc}
\hline Case & Age (d) & BW $(\mathbf{k g})$ & $\begin{array}{c}\text { AV/MV } \\
\text { lesions }\end{array}$ & $\begin{array}{c}\text { Diameter of } \\
\text { AscAo (mm) }\end{array}$ & Pulmonary type & $\begin{array}{c}\text { Blood source } \\
\text { size (mm) }\end{array}$ & Result \\
\hline 1 & 11 & 2.2 & A/A & 3.0 & B-T & 3.0 & Survived \\
2 & 20 & 2.6 & A/A & 1.5 & B-T & 3.0 & Operative death \\
3 & 30 & 1.5 & A/A & 2.0 & B-T & 3.5 & Survived \\
4 & 15 & 2.6 & A/S & 3.5 & B-T & 3.5 & Survived \\
5 & 26 & 1.7 & A/S & 3.0 & RV-PA & 5.0 & Survived \\
6 & 25 & 3.2 & S/S & 6.0 & RV-PA & 6.0 & Survived \\
7 & 25 & 3.2 & A/A & 1.0 & RV-PA & 6.0 & Survived \\
8 & 7 & 3.0 & A/A & 2.5 & RV-PA & 6.0 & Survived \\
9 & 13 & 2.6 & A/A & 2.0 & RV-PA & 6.0 & Operative death \\
10 & 2 & 2.8 & A/A & 2.0 & RV-PA & 6.0 & Operative death \\
\hline
\end{tabular}

$A V$, Aortic valve; $M V$, mitral valve; $A$, atresia; $S$, stenosis; $A s c A o$, ascending aorta; $B$-T, Blalock-Taussig shunt; $R V$ - $P A$, right ventricle-pulmonary artery conduit.

notomy, an expanded polytetrafluoroethylene (E-PTFE) graft, 3.0 or $3.5 \mathrm{~mm}$ in diameter, was sewn to the innominate artery. CPB was commenced with bicaval venous cannulation and with the E-PTFE graft at the innominate artery as an arterial line. The descending thoracic aorta was identified through an incision in the posterior pericardium just superior to the diaphragm. An angled metal-tipped cannula $(2.1 \mathrm{~mm}$ in outer diameter; Japan Medical Supply Co, Hiroshima, Japan) was inserted through a purse-string suture placed in the descending aorta. Thereafter, extracorporeal circulation with single pump and double arterial lines was established (Figure 1). Pump flow was maintained at 150 to $180 \mathrm{~mL} \cdot \mathrm{min}^{-1} \cdot \mathrm{kg}^{-1}$, and the lowest rectal temperature was from $29^{\circ} \mathrm{C}$ to $31^{\circ} \mathrm{C}$. The atrial septal defect was enlarged in all cases. In the arch reconstruction of the last 9 cases, coarctectomy and subsequent direct anastomosis of the arch and the descending aorta were performed maintaining CPB flow to the whole body including coronary flow. Then, the main pulmonary artery (PA) was transected. After cardioplegic arrest was obtained, the ascending aorta was incised longitudinally from the transverse arch down to the diminutive sinus of Valsalva (Figure 2). The neoaorta was constructed by anastomosing the main PA to the ascending aorta and transverse arch with the use of continuous absorbable polydioxanone suture (PDS 6-0; Ethicon, Inc, Somerville, NJ). In the first case of the series, an autologous pericardial roll was interposed between the main PA and the aortic arch. After completion of the neoaorta and the aortic arch, perfusion through the innominate artery was ceased and the whole body was perfused with the single cannula in the descending aorta. In the first 4 cases, the source of pulmonary blood flow was a systemic-pulmonary shunt. The E-PTFE graft used for cerebral perfusion was subsequently anastomosed to the right PA to act as a shunt in these patients. In the remaining 6 patients, an E-PTFE graft was interposed between the right ventricle (RV) and the distal PA (RV-PA conduit) for the pulmonary blood source (Figure 3). The RV-PA conduit was constructed by means of an incision on the RV free wall about $15 \mathrm{~mm}$ below the origin of the main PA and equally apart from the right and the anterior descending coronary arteries. A small piece of muscle was excised to create an unobstructed opening. The size of the graft used was $5 \mathrm{~mm}$ for patients with a body weight less than 2.5 $\mathrm{kg}$ and $6 \mathrm{~mm}$ for heavier patients. CPB time was 127 to 217 minutes (178 \pm 33 minutes), and aortic crossclamp time was 32 to 78 minutes (56 \pm 17 minutes). Circulatory arrest was completely avoided.

\section{Results}

Three patients died early postoperatively (Table 1). One patient died of intraoperative massive airway bleeding. The second patient was in shock preoperatively and died of sepsis on the 29th postoperative day. The third patient with aortic atresia had preoperative shock, and she had an extraordinarily large left ventricular mass that almost obliterated the cavity, which must have had deleterious effects on RV function. Communications from the left ventricular cavity to the coronary arteries had been recognized during preoperative echocardiography. She died of ventricular dysfunction and hypoxia 3 hours after the operation. The first patient had a conventional systemic-pulmonary shunt and the latter 2 patients had an RV-PA conduit. Operative methods used had no bearing on the cause of death. Late death occurred in 2 patients with a systemic-pulmonary shunt, 3 and 4 months after the operation, respectively. One patient died of intracranial hemorrhage caused by trauma. Cause of death in another patient was unknown. All 5 longterm survivors underwent a bidirectional Glenn procedure, and 4 of them later had a Fontan operation. Another patient is awaiting a Fontan operation.

A pediatric neurologist checked 7 operative survivors, and seizure or other neurologic deficit was not seen. We are not routinely taking electroencephalograms or computed tomograms of the brain, but the electroencephalograms performed in 3 patients showed no abnormalities. Complications derived from cannulation of the descending aorta or innominate artery perfusion were not encountered. Peritoneal dialysis was performed in 4 patients with the aim of maintaining water and electrolyte balance. Actually, urine output during the first 24 hours was from 183 to $365 \mathrm{~mL}(227 \pm 94 \mathrm{~mL})$, and the postoperative maximum serum creatinine level was 0.7 to $1.0 \mathrm{mg} / \mathrm{dL}$. Mechanical ventilation time was $128 \pm 61$ hours including the average interval of 72 hours for delayed sternal closure, which is our routine postoperative protocol. Postoperative cardiac catheterization was performed in 7 


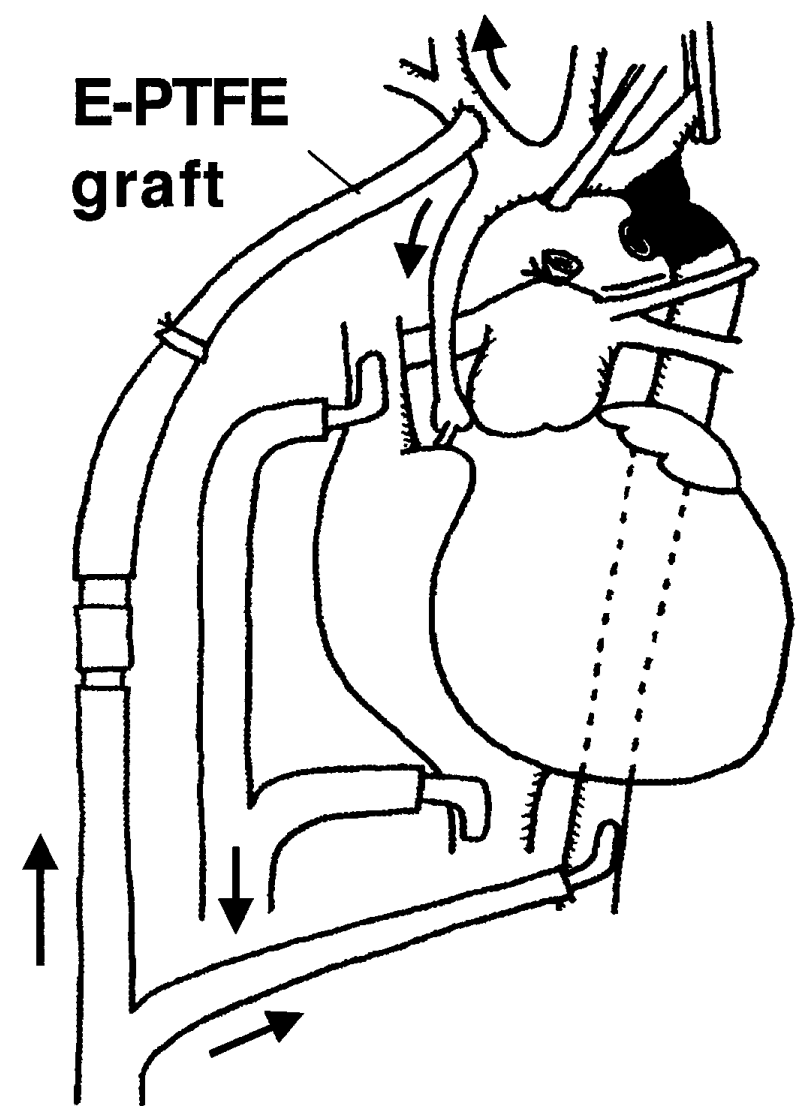

Figure 1. The CPB circuit used in this series. The brain was perfused with the E-PTFE graft anastomosed to the innominate artery, and the lower part of the body was perfused throughout the surgical procedure with an arterial cannula inserted in the descending aorta. Coronary flow was maintained through the retrograde aortic flow from the innominate artery during the coarctectomy and end-to-end anastomosis.

patients at an interval of 2 months postoperatively, and there was no significant stenosis in the neoaorta or reconstructed aortic arch. Coronary flow was nonobstructive in all patients, and the pulmonary blood flow was also good with either type of pulmonary blood source. Long-term RV function after the RV-PA conduit procedure was a matter of concern. Cardiac catheterization after the Fontan operation in 4 patients (mean follow-up time $24.0 \pm 2.6$ months), except the 2 who died early, showed a cardiac index of 3.5 to $4.1 \mathrm{~L}$ $\cdot \min ^{-1} \cdot \mathrm{kg}^{-1}\left(3.7 \pm 0.3 \mathrm{~L} \cdot \mathrm{min}^{-1} \cdot \mathrm{kg}^{-1}\right)$ and an $\mathrm{RV}$ ejection fraction of $50 \%$ to $65 \%(60 \% \pm 7 \%)$. Tricuspid regurgitation was mild in 1 patient and trivial in the others.

\section{Comment}

In the Norwood procedure, circulatory arrest with deep hypothermia is commonly used. However, this technique has some risks, such as neurologic deficits and renal failure, especially when the circulatory arrest time becomes longer.
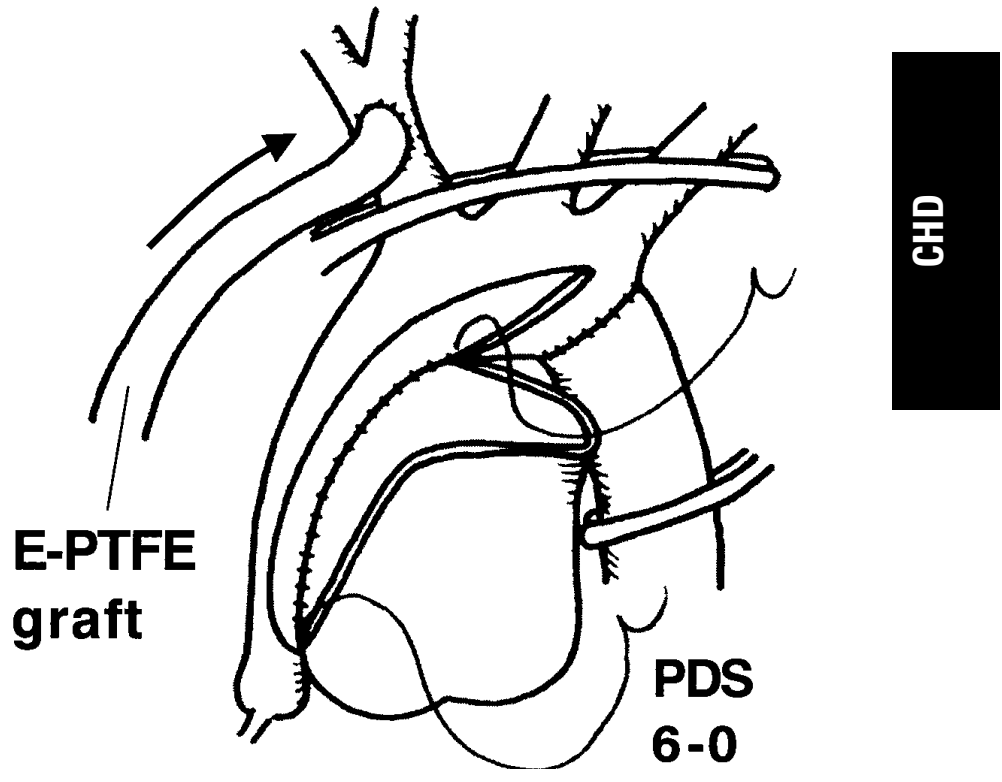

Figure 2. Construction of the neoaorta. The proximal stump of the main PA was anastomosed with the ascending aorta and the transverse arch without patching material. Perfusion of the brain and the lower part of the body was maintained during this procedure. PDS, Polydioxanone suture.

$\mathrm{We}^{7}$ reported earlier a technique for lower body perfusion in which a synthetic graft is anastomosed to the descending aorta through a left thoracotomy in primary repair of interrupted aortic arch. This technique combined with innominate artery perfusion could completely avoid circulatory arrest throughout the operation. However, a left thoracotomy seemed too invasive for neonates in critical condition. Therefore, we adopted cannulation of the descending aorta just superior to the diaphragm through a median sternotomy in the Norwood procedure, which made additional thoracotomy unnecessary. Clinical seizure and manifestations of other neurologic deficits were not seen among the survivors, and the postoperative course was stable with satisfactory renal function. Avoidance of circulatory arrest also made deep hypothermia unnecessary in our CPB technique. Postoperative severe coagulopathy and capillary leakage syndrome, which are known as adverse effects of deep hypothermia, were not encountered among the survivors.

In addition to the new CPB technique, we adopted a new surgical technique in the present series, that is, a nonvalved RV-PA conduit. It was first applied clinically by Norwood and his colleagues ${ }^{8}$ using a valved or nonvalved conduit. Recently in Japan, Kishimoto and coworkers ${ }^{9}$ reevaluated the method and found that the postoperative circulation was very stable after this procedure. In comparing the postoperative clinical course of the patients with a systemic-pulmonary shunt and that of patients with an RV-PA conduit in our series, 


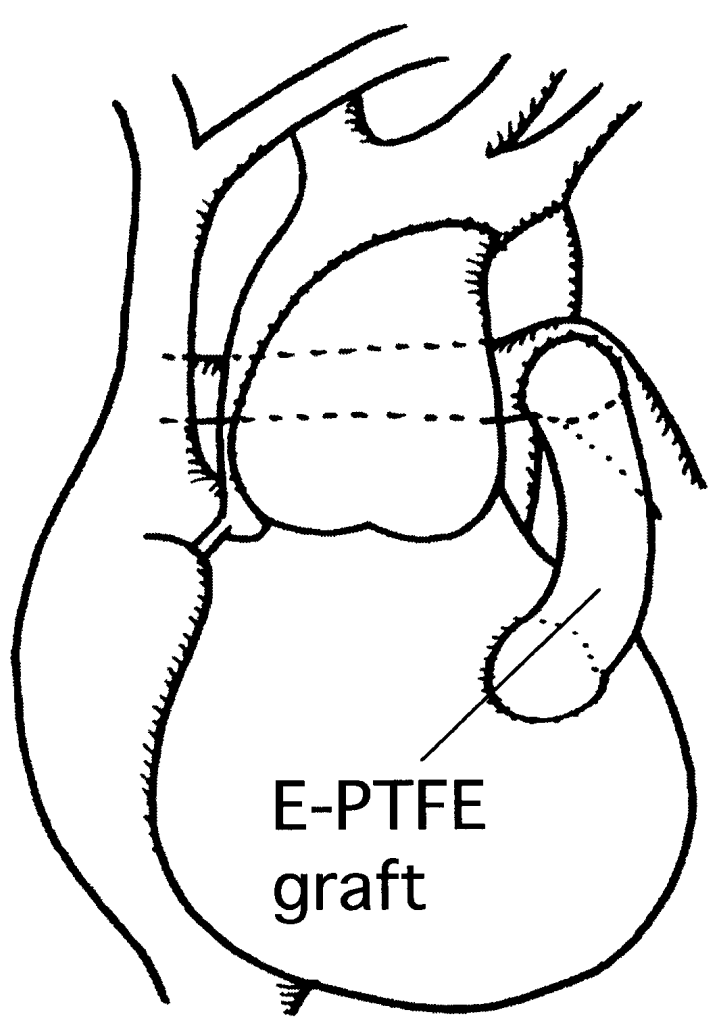

Figure 3. Modification of the Norwood procedure with the use of an RV-PA conduit instead of a systemic-pulmonary shunt. A small incision was made in the avascular area of the RV wall, and the EPTFE graft was interposed between the incision and the distal PA.

we found the stability of circulation in terms of systemic blood pressure or arterial oxygen saturation to be superior in the patients with an RV-PA conduit. We think that the higher systemic diastolic pressure in the patients with an RV-PA conduit leads to better coronary blood flow and results in stable circulation (Figure 4). On the contrary, a decrease in the systemic blood pressure in patients with a systemic-pulmonary shunt causes coronary hypoperfusion and ventricular dysfunction, which may further decrease the blood pressure; thus, a vicious cycle easily develops. RV dysfunction derived from ventriculotomy was our concern, but no such ill effect has been demonstrated so far, although long-term follow-up is needed.

In conclusion, the Norwood procedure for hypoplastic left heart syndrome was successfully accomplished with complete avoidance of deep hypothermic circulatory arrest by cannulation of the descending aorta combined with cerebral perfusion through the innominate artery. A conduit between the RV and the PA seems an excellent alternative

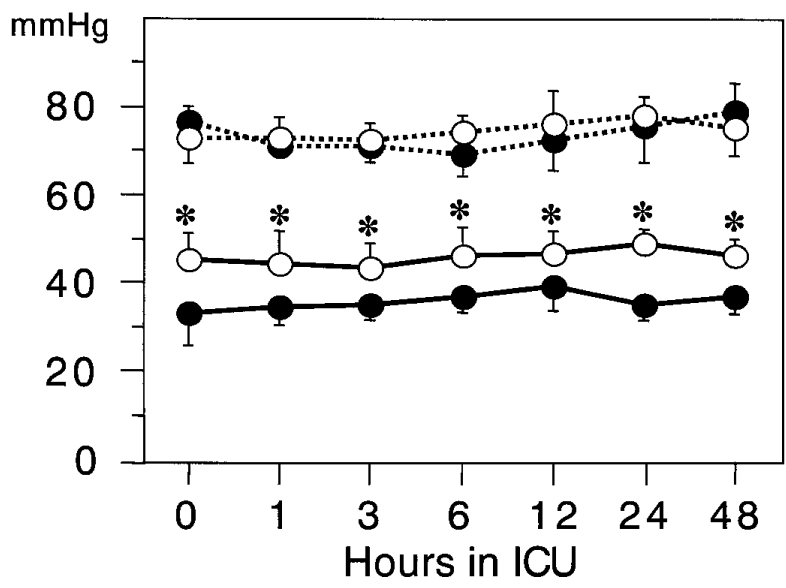

Figure 4. Comparison of the postoperative systemic arterial pressure in patients with a conventional Blalock-Taussig shunt $(n=3$, closed circles) and with an RV-PA conduit ( $n=5$, open circles). The systolic pressure (broken line) and the diastolic pressure (solid line) are shown (mean \pm standard deviation). Statistical significance was recognized in the differences in the diastolic pressures in the 2 groups $\left({ }^{*} P<.05\right.$, unpaired Student $t$ test). There was no significant difference in the systolic pressures in the 2 groups. ICU, Intensive care unit.

pulmonary blood source, although deleterious effects of right ventriculotomy need to be carefully monitored.

We thank Dr O. P. Yadava, Sir Ganga Ram Hospital, for his valuable comments on the manuscript.

\section{References}

1. Rigden SP, Barratt TM, Dillon MJ, Kind PR, de Leval M, Stark J. Acute renal failure complicating cardiopulmonary bypass surgery. Arch Dis Child. 1982;57:425-30.

2. Asfour B, Bruker B, Kehl HG, Frund S, Scheld HH. Renal insufficiency in neonates after cardiac surgery. Clin Nephrol. 1996;46:59-63.

3. Bellinger DC, Jonas RA, Rappaport LA, Wypij D, Wernovsky G, Kuban KCK, et al. Developmental and neurologic status of children after heart surgery with hypothermic circulatory arrest or low-flow cardiopulmonary bypass. N Engl J Med. 1995;332:549-55.

4. Miller G, Tesman JR, Ramer JC, Baylen BG, Myers JL. Outcome after open-heart surgery in infants and children. J Child Neurol. 1996;11:49-53.

5. Oates RK, Simpson JM, Turnbull JAB, Cartmill TB. The relationship between intelligence and duration of circulatory arrest with deep hypothermia. J Thorac Cardiovasc Surg. 1995;110:786-92.

6. Imoto Y, Kado H, Shiokawa Y, Fukae K, Yasui H. Norwood procedure without circulatory arrest. Ann Thorac Surg. 1999;68:559-61.

7. Yasui H, Kado H, Yonenaga K, Kawasaki S, Shiokawa Y, Kouno H, et al. Revised technique of cardiopulmonary bypass in one-stage repair of interrupted aortic arch complex. Ann Thorac Surg. 1993;55:1166-71.

8. Norwood WI, Lang P, Castaneda AR, Campbell DN. Experience with operation for hypoplastic left heart syndrome. $J$ Thorac Cardiovasc Surg. 1981;82:511-9.

9. Kishimoto H, Kawahira Y, Kawata H, Miura T, Iwai S, Mori T. The modified Norwood procedure on a beating heart. J Thorac Cardiovasc Surg. 1999;118:1130-2. 